$$
\text { CONF-9609168--2 }
$$

Invited Session

International Conference on Physics of Transition Metals, Osaka, Japan, September $23-27,1996$

\title{
SPIN AND CHARGE DYNAMICS OF CHROMIUM ALLOYS
}

R. S. Fishman, V. S. Viswanath, and S. H. Liu

\begin{abstract}
-The submitted manuscript has been authored by a contractor of the U.S. Government under contract No. DE-AC05-960R24464.

Accordingly, the U.S. Government retains a nonexclusive, royalty-free license to publish or

reproduce the published form of this

contribution, or allow others to do so, for U.S.

Government purposes."
\end{abstract}

\author{
SOLID STATE DIVISION \\ OAK RIDGE NATIONAL LABORATORY \\ Managed by \\ LOCKHEED MARTIN ENERGY RESEARCH CORP. \\ Under \\ Contract No. DE-AC05-96OR22464 \\ With the \\ U. S. DEPARTMENT OF ENERGY \\ OAK RIDGE, TENNESSEE
}

July 1996 


\section{DISCLAIMER}

Portions of this document may be illegible in electronic image products. Images are produced from the best available original document. 


\title{
SPIN AND CHARGE DYNAMICS OF CHROMIUM ALLOYS
}

\author{
by
}

\author{
R.S. Fishman and V.S. Viswanath
}

Solid State Division, P.O. Box 2008, Oak Ridge National Lab, Oak Ridge, TN 37831-6032

and

S.H. Liu

Physics Department, University of California, San Diego, CA 92093

\begin{abstract}
Both the spin- and charge-density waves of $\mathrm{Cr}$ alloys are produced by the Coulomb attraction between electrons and holes on nearly-nested Fermi surfaces. Driven by quasiparticle transitions, transverse spin-wave and longitudinal phason modes are associated with the rotational and translational symmetries of pure $\mathrm{Cr}$ and its dilute alloys. At low frequencies, both spin and charge phasons have a nearly linear dispersion with a mode velocity which approaches the spin-wave velocity as $T$ approaches $T_{N}$ or as the mismatch between the Fermi surfaces increases.
\end{abstract}

\section{DISCLAIMER}

This report was prepared as an account of work sponsored by an agency of the United States Government. Neither the United States Government nor any agency thereof, nor any of their employees, makes any warranty, express or implied, or assumes any legal liability or responsibility for the accuracy, completeness, or usefulness of any information, apparatus, product, or process disclosed, or represents that its use would not infringe privately owned rights. Reference herein to any specific commercial product, process, or service by trade name, trademark, manufacturer, or otherwise does not necessarily constitute or imply its endorsement, recommendation, or favoring by the United States Government or any agency thereof. The views and opinions of authors expressed herein do not necessarily state or reflect those of the United States Government or any agency thereof. 
Due to their relatively small densities-of-states and the nearly perfect nesting of their Fermi surfaces, ${ }^{1,2} \mathrm{Cr}$ and $\gamma-\mathrm{Mn}$ alloys are the only transition metals that become antiferromagnetic. In $\gamma-\mathrm{Mn}$ alloys, the nested electron and hole Fermi surfaces are close enough in size that the spin-density wave (SDW) is always commensurate (C) with the lattice. So pure $\mathrm{Cr}$ and its dilute alloys ${ }^{3}$ are the only transition metals with an incommensurate (I) SDW. Shortly after the discovery of SDW magnetism in pure $\mathrm{Cr}$, experimentalists ${ }^{4}$ also detected a charge-density wave (CDW) with twice the wavevector and half the period of the SDW. Although many experiments have been performed on the spin excitations ${ }^{5-8}$ of pure $\mathrm{Cr}$, it has only now become feasible to measure the charge excitations about the ordered CDW. Recently, Fishman and $\mathrm{Liu}^{9}$ used the random-phase approximation (RPA) to study the spin excitations of pure $\mathrm{Cr}$ and its dilute alloys. In this paper, we review that work and present the first theoretical results for the charge dynamics of I Cr alloys.

In order to minimize the condensation free energy on both sides of the two nested Fermi surfaces ${ }^{10}$ of $C_{r}$ alloys, the ordering wavevectors $\mathbf{Q}_{ \pm}^{\prime}=(\mathbf{G} / 2)\left(1 \pm \partial^{\prime}\right)$ of the SDW lie slightly closer to $G / 2$ than the nesting wavevectors. The CDW wavevectors ${ }^{11}$ are then given by $2 \mathbf{Q}_{ \pm}^{\prime}$, on either side of the reciprocal lattice vector $\mathbf{G}=4 \pi \hat{\mathbf{z}} / a$ where $a$ is the lattice constant of the conventional unit cell. For pure $\mathrm{Cr}, \partial^{\prime} \approx 0.04$ so that the wavelength ${ }^{3}$ of the SDW is about 25 lattice constants. With V doping, the hole surface becomes even larger and $\partial^{\prime}$ increases; with $\mathrm{Mn}$ or Fe doping, the electron surface grows and $\partial^{\prime}$ decreases. When the Mn concentration exceeds $0.3 \%$ or the Fe concentration exceeds $2.4 \%$, the SDW becomes commensurate with $\partial^{\prime}=0$ and $\mathbf{Q}_{ \pm}^{\prime}=\mathbf{G} / 2$.

If the momenta of the holes are translated by the nesting wavevectors, ${ }^{10,11}$ the mismatch between the electron and hole energies is defined by $z_{0}$. This mismatch energy increases linearly with the $\mathrm{V}$ concentration and decreases linearly with the concentration of $\mathrm{Mn}$ or Fe. The scale for both $z_{0}$ and the excitation frequency $\omega$ is set by the Néel temperature $T_{N}^{*} \approx 80 \mathrm{meV}$ of a perfectly nested alloy with $z_{0}=0$. 
Introducing the periodic Bloch functions $u(\mathbf{r})$ of the d-band electrons normalized in volume $V$, the SDW and CDW can be written as ${ }^{12}$

$$
\begin{gathered}
\mathbf{S}(\mathbf{r})=\alpha_{s} V \rho_{e h} \frac{g(T)}{\lambda} \hat{\mathbf{m}}|u(\mathbf{r})|^{2} \cos \left(\frac{1}{2}\left(\mathbf{Q}_{+}^{\prime}+\mathbf{Q}_{-}^{\prime}\right) \cdot \mathbf{r}-\phi\right) \cos \left(\frac{1}{2}\left(\mathbf{Q}_{+}^{\prime}-\mathbf{Q}_{-}^{\prime}\right)-\frac{\theta}{2}\right), \\
\varrho(\mathbf{r})=-\alpha_{c} V \rho_{e h} \frac{\delta(T)}{\lambda^{\prime}}|u(\mathbf{r})|^{2} \cos \left(\left(\mathbf{Q}_{+}^{\prime}-\mathbf{Q}_{-}^{\prime}\right) \cdot \mathbf{r}-\theta\right)
\end{gathered}
$$

where $\alpha_{s}$ and $\alpha_{c}$ are positive constants, $\phi$ and $\theta$ are arbitrary angles, $\rho_{e h}$ is the densityof-states for both spins, and $\hat{\mathbf{m}}$ is the spin polarization direction. In addition, $\lambda$ and $\lambda^{\prime}$ are dimensionless Coulomb constants which parametrize ${ }^{13}$ the interaction between the electrons and holes. Both the SDW and CDW order parameters $g(T)>0$ and $\delta(T)<0$ vanish above $T_{N}$. Since

$$
\mathbf{Q}_{+}^{\prime}-\mathbf{Q}_{-}^{\prime}=2 \mathbf{Q}_{+}^{\prime}-\mathbf{G}=\mathbf{G}-2 \mathbf{Q}_{-}^{\prime},
$$

the CDW is the second harmonic ${ }^{11}$ of the SDW. Eqs.(1) and (2) also imply that the SDW and CDW are in phase: the electron number $\varrho(\mathbf{r})$ is largest whenever the magnitude $|\mathbf{S}(\mathbf{r})|$ is a maximum.

Since the Bloch functions and density waves are strongly peaked ${ }^{14,15}$ at the atomic sites, we may replace

$$
|u(\mathbf{r})|^{2} \rightarrow \frac{1}{N} \sum_{\mathbf{R}} \delta(\mathbf{r}-\mathbf{R}),
$$

where the sum runs over the $N$ lattice sites at positions $\mathbf{R}$. Then the maximum values of the spin and electron number at any lattice site in the I phase are $S_{0}=(V / N) \rho_{\text {eh }} \alpha_{s} g / \lambda$ and $\varrho_{0}=-(V / N) \rho_{e h} \alpha_{c} \delta / \lambda^{\prime}$.

The amplitude $\varrho_{0}$ of the CDW is controlled ${ }^{12}$ by the dimensionless parameter $\lambda^{\prime}$, which lies between 0 and $1 / 2$. In the limit $\lambda^{\prime} \rightarrow 0, \delta$ also vanishes but the ratio $\delta / \lambda^{\prime}$ approaches a small nonzero value which depends on the energy mismatch $z_{0}$. As $\lambda^{\prime} \rightarrow 1 / 2$, 
the I phase becomes unstable for all $z_{0}$. Charge conservation in the $\mathrm{C}$ phase requires that the CDW must vanish so that $\theta=\pi / 2$ plus some multiple of $\pi$.

Spin and charge excitations with frequency $\omega$ and momentum $q$ away from one of the two I ordering wavevectors are driven by quasiparticle transitions ${ }^{9}$ with energy change $\omega$ and momentum change $q$. The complexity of the quasiparticle energies creates a rich spectrum of spin and charge excitations, which we have evaluated within the RPA. Despite the simplicity of the RPA for a Heisenberg antiferromagnet, the three bands of quasiparticles create formidable technical difficulties for an itinerant antiferromagnet.

The low-frequency Goldstone modes of $\mathrm{I} \mathrm{Cr}$ alloys are associated with the invariance of the free energy under translations and rotations of the density waves in Eqs.(1) and (2). While spin-wave (SW) modes are associated with the free energy's invariance under rotations of the polarization direction $\hat{\mathbf{m}}$, phason modes are associated with its invariance under translations of the phase angle $\theta$. Both the SW's and the spin phasons evolve from the SDW ordering wavevectors $\mathbf{Q}_{ \pm}^{\prime}+\boldsymbol{\Upsilon}$ for any reciprocal lattice vector $\boldsymbol{Y}$; the charge phasons evolve from the CDW ordering wavevectors $2 \mathbf{Q}_{ \pm}^{\prime}+\mathbf{\Upsilon}$.

Unlike the SW and phason modes, two other classes of excitations ${ }^{9}$ are not associated with symmetries of the free energy. Amplitude modes are produced by oscillations of the SDW and CDW amplitudes about their equilibrium values. An unexpected class of collective modes dubbed wavons is associated with oscillations of the SDW wavevectors $\mathbf{Q}_{ \pm}^{\prime}$. Since neither amplitudons nor wavons are Goldstone modes, their frequencies do not vanish as their wavelengths diverge. So uniform changes in the density-wave amplitudes or wavevectors cost a nonzero energy. The following discussion concentrates on the Goldstone modes introduced above.

For simplicity, we have set $\lambda^{\prime}=0$ in our study of the spin dynamics. With other parameters typical of pure $\mathrm{Cr}$ with $T / T_{N}=0.5$, the spin excitations are plotted in Fig.1. 
The SW modes have a linear dispersion with the same mode velocity $c=v_{F} / \sqrt{3} \approx 1500$ $\mathrm{meV} \dot{A}$ as in the $\mathrm{C}$ regime. Because this mode velocity is so large - about 50 times the SW velocity of a rare-earth antiferromagnet - the splitting of each satellite into side peaks is difficult to observe using neutrons. Although $c$ is independent of temperature, the integrated weight ${ }^{9} s_{t} \propto(n+1) g^{2} / \lambda^{2} \omega$ of each SW mode vanishes as $T$ approaches $T_{N}$ and decreases with increasing frequency. Here, $n=1 /(\exp (3 \omega)-1)$ is the Boltzmann factor. Just as in a Heisenberg antiferromagnet, the SW's correspond to the rigid rotations of the spins about their equilibrium positions.

Spin phasons are produced by the dynamics of the phase $\theta$ in Eq.(1). For small frequencies, the phasons evolve linearly with mode velocity $c_{p h}<c$. As $T$ approaches $T_{N}$ or as the mismatch energy $z_{0}$ increases, $c_{p h}$ approaches $c$. The spin phason velocity in Fig. 1 is about $0.65 c$. At low frequencies, the integrated strengths $s_{l}$ of the spin phasons is also proportional to $(n+1) g^{2} / \lambda^{2} \omega$ and also diverges like $1 / \omega^{2}$ as $\omega \rightarrow 0$. Because the strength of any excitation is inversely proportional to its slope $d \omega / d q$, the intersection of the inner phason modes with G/2 generates a strongly enhanced cross section which was recently observed by Fukuda et al. ${ }^{8}$ The inner phasons also bend the SW cones towards G/2, as first observed by Fincher et al. ${ }^{5}$

With the additional complexity of two Coulomb constants $\lambda$ and $\lambda^{\prime}$ instead of one. the charge dynamics may also be studied within the RPA. ${ }^{16}$ The charge phasons are plotted in Fig.2 using a coupling constant $\lambda^{\prime}=0.15$. With other parameters close to those in Fig.1, the CDW amplitude $\varrho_{0}$ lies between 0.0083 and 0.0128 electrons per site, depending on the density-of-states. As shown, the charge phason velocity $c_{p h}^{\prime}$ is about $0.67 c$. Since the spin excitation spectrum of Fig.1 was obtained with $\lambda^{\prime}=0$, the velocities of the spin and charge phasons cannot be directly compared. The charge phason velocity $c_{p h}^{\prime}$ increases with increasing temperature or energy mismatch and with decreasing coupling $\lambda^{\prime}$. For $z_{0} / T_{N}^{*}=6.75$ and $T / T_{N}=0.5$, we find that $c_{p h}^{\prime} / c \approx 0.94$. Like the strengths $s_{t}$ and $s_{l}$ of 
the spin excitations, the integrated strength $s_{l}^{\prime} \propto(n+1) \delta T_{N}^{*} / \omega$ of the charge phasons also diverges like $1 / \omega^{2}$ as $\omega \rightarrow 0$.

For frequencies above about $0.05 T_{N}^{*} \approx 4 \mathrm{meV}$, the charge excitation spectrum contains six peaks: two on either side of each satellite wavevector $2 Q_{ \pm}^{\prime}$ and two more on eitber side of G. With increasing energy, the inner four charge phasons dominate the outer two phasons, the CDW satellites will shift inwards towards G, and the inelastic scartering peaks near $G$ will move apart. For frequencies below $67 \mathrm{meV}$, the inner phason branches evolving from $\mathbf{Q}_{+}^{\prime}$ or $\mathbf{Q}_{-}^{\prime}$ are associated with two peaks which approach each other with increasing energy. The maximum in the phason frequency near $67 \mathrm{meV}$ should produce a large peak in the cross section at a wavevector close to $\mathbf{G}\left(1 \pm \partial^{\prime}\right)$, midway between $2 \mathbf{Q}_{ \pm}^{\prime}$ and $\mathbf{G}$.

The predictions of our work on the incommensurate spin dynamics should be straightforward to test experimentally. With $\mathrm{V}$ doping, the spin phason velocity $c_{p h}$ of $\mathrm{Cr} V$ alloys will increase and the phasons will intersect $q=0$ at a higher energy. So at low temperatures, the $60 \mathrm{meV}$ peak in the $\mathbf{G} / 2$ cross section should move to higher energies.

Our model for the charge phasons is unlikely to work below about $10 \mathrm{mel}$. First, the pinning of the CDW by lattice defects will break the translational symmetry of the free energy and introduce an energy gap into the CDW excitation spectrum. Second, the coupling between the CDW and strain wave will hybridize the CDW phasons and lattice phonons at low frequencies. So only above about $10 \mathrm{meV}$ can inelastic scattering be used to study the charge phasons.

Due to the coupling between the SDW and CDW order parameters $g$ and $\delta \propto \lambda^{\prime} g^{2} / T_{N}^{*}$, charge phasons may be probed using either x-rays or neutrons. In a neutron-scattering experiment, longitudinal spin fluctuations couple indirectly to the CDW phase $\theta$ through the phase $\theta / 2$ of the SDW. Compared to the SDW strength, the strength of the charge 
phasons is given by $s_{l}^{\prime} / s_{t} \propto \lambda^{\prime} \lambda^{2}$. Unfortunately, the dimensionless coupling constant $\lambda<1$ is undetermined by our model. For $\lambda^{\prime}=0.15$ and a reaconable estimate of $\lambda=0.2$, the charge phasons would be roughly 160 times weaker than the SW modes at low frequencies. So except near the maximum phason frequency of about $67 \mathrm{meV}$, the charge phasons may not be observable using neutrons.

A direct measurement of the charge phasons using $x$-rays may also be difficult. Relative to the Bragg peak, the inelastic $\mathrm{x}$-ray scattering strength is given by

$$
\frac{S_{l}^{\prime}}{S_{G}} \propto \lambda^{\prime} \frac{n+1}{f^{2}} \frac{\omega}{T_{N}^{*}}\left(\frac{g}{T_{N}^{*}}\right)^{2}\left(\frac{V}{N}\right)^{2}\left(\frac{T_{i}^{*}}{v_{F}}\right)^{3} \rho_{e h} \Delta \omega_{r}
$$

where $f=24$ is the number of electrons on a $\mathrm{Cr}$ atom and $\Delta \omega_{r}$ is the instrumental resolution. For the parameters in Fig. $2, \omega=0.5 T_{N}^{*}$, and a resolution of $\Delta \omega_{r}=30 \mathrm{meV}$, $S_{l}^{\prime} / S_{G}$ is of order $10^{-10}$. With newly available and improved synchrotron sources, this relative strength may be just large enough to observe the charge excitations.

Using either technique, the peak in the cross section near $67 \mathrm{meV}$ for pure $\mathrm{Cr}$ will shift upwards with V doping, as the charge phason velocity increases. However, the location of this peak cross section in momentum space should be relatively insensitive to doping. Since the $\mathrm{CDW}$ is enhanced by doping ${ }^{12}$ with $\mathrm{Fe}$, the chances of detecting charge excitations may be increased by performing inelastic measurements on $\mathrm{CrFe}$ alloys.

One of us (RF) would like acknowledge support from the U.S. Department of Energy under Contract No. DE-FG06-94ER45519 and under Contract No. DE-AC0584OR21400 with Martin Marietta Energy Systems, Inc. SL would like to thank Profs. L. Sham and R. Dynes for their hospitality at UCSD. Useful conversations with B. Larson are also gratefully acknowledged. 


\section{REFERENCES}

1. W.M. Lomer, Proc. Phys. Soc. London 80, 489 (1962).

2. P.A. Fedders and P.C. Martin, Phys. Rev. 143, 8245 (1966).

3. For comprehensive reviews of $\mathrm{Cr}$ alloys, see E. Fawcett, Rev. Mod. Phys. 60, 209 (1988) and E. Fawcett, H.L. Alberts, V. Yu. Galkin, D.R. Noakes, and J. V. Yakhmi, Rev. Mod. Phys. 66, 26 (1994).

4. Y. Tsunoda, M. Mori, N. Kunitomi, Y. Teraoka, and J. Kanamori, Sol. State Commun. 14, 287 (1974).

5. C.R. Fincher, G. Shirane, and S.A. Werner, Phys. Rev. B 24, 1312 (1981).

6. S.K. Burke, W.G. Stirling, K.R.A. Ziebeck, and J.G. Booth, Phys. Rev. Lett. 51, 494 (1983).

7. J.E. Lorenzo, B.J. Sternlieb, G. Shirane, and S.A. Werner, Phys. Rev. Lett. 72, 1762 (1994).

8. T. Fukuda, Y. Endoh, K. Yamada, M. Takeda, S. Itoh, M. Arai, and T. Otomo, (preprint).

9. R.S. Fishman and S.H. Liu, Phys. Rev. Lett. 76, 2398 (1996); (unpublished).

10. R.S. Fishman and S.H. Liu, Phys. Rev. B 48, 3820 (1993).

11. C.Y. Young and J.B. Sokoloff, J. Phys. F 4, 1304 (1974).

12. X.W. Jiang and R.S. Fishman, (unpublished).

13. A.W. Overhauser, Phys. Rev. 128, 1437 (1962); Phys. Rev. 167, 691 (1968); Phys. Rev. B 3, 3173 (1971).

14. M. Mori and Y. Tsunoda, J. Phys. C 5, L77 (1993).

15. R.M. Moon, W.C. Koehler, and A.L. Trego, J. Appl. Phys. 37, 1036 (1966).

16. V.S. Viswanath and R.S. Fishman, (unpublished). 


\section{FIGURE CAPTIONS}

1. The spin excitation frequency $\omega$ versus wavevector $q$ for the transverse $S W$ (dashed) and longitudinal phason (solid) modes with $z_{0} / T_{N}^{*}=4.7, T / T_{N}=0.5$, and $\lambda^{\prime}=0$.

2. The charge phason frequency (solid) versus wavevector $q$ with $z_{0} / T_{N}^{*}=5.0, T / T_{N}=$ 0.5 , and $\lambda^{\prime}=0.15$. The dashed lines with slopes $\pm c$ provide a guide to the eye and are not associated with charge excitations. 


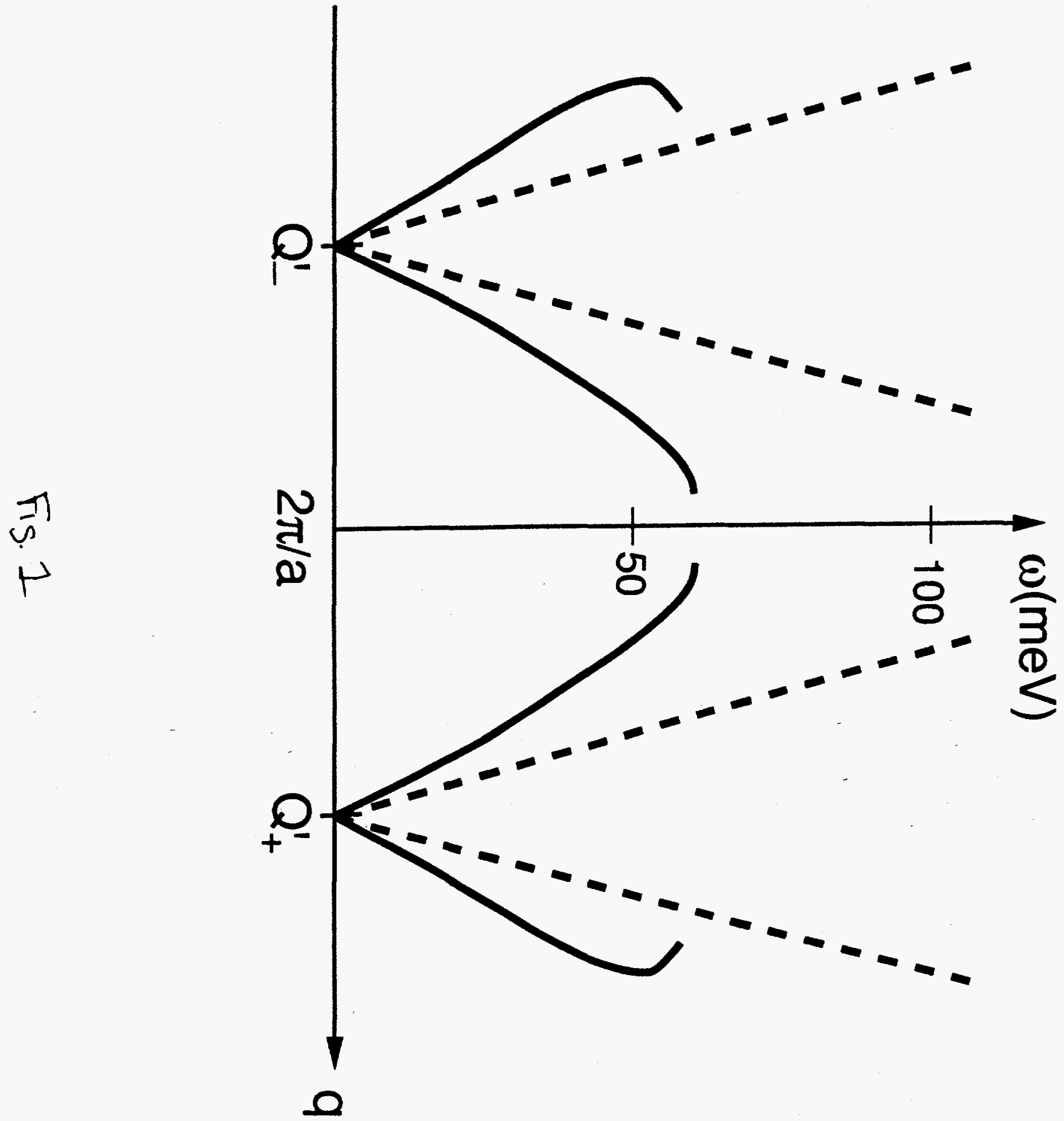




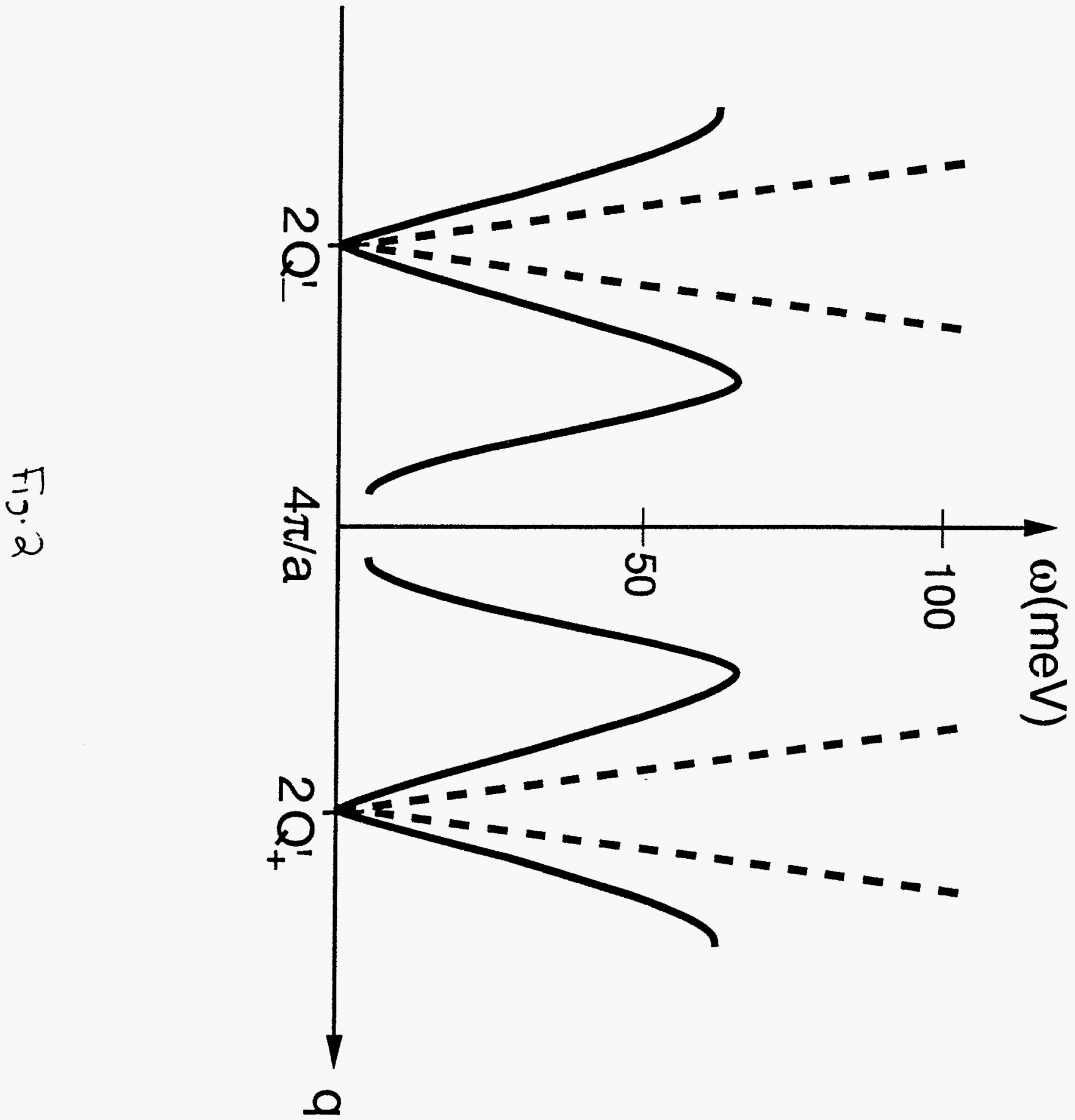

